litersystem „Eppendorf“ auskommt. Weiterhin hat sie den Vorzug, daß der von ihr bestrichene Konzentrationsbereich größer ist. Als Nachteil ist zu erwähnen, $\mathrm{da} \beta$ der interessierende Konzentrationsbereich $(90$ bis $120 \mathrm{mVal}$ ) nicht soweit gespreizt ist wie bei der Methode am PF 5-Gerät, wo dieser Bereich die ganze Skala ein- nimmt. Weiterhin ist es aufgrund der etwas geringeren Nachverdünnung nicht so leicht, erforderlichenfalls zu noch kleineren Serum-Mengen überzugehen.

Für die technische Hilfe danken wir (BechtLer) Frl. Siebert u. Frl. Dahlmann in Hamburg.

\title{
Literatur
}

1. Lang, W., diese Z. 3, 186 (1965). - 2. Lang, W., Der optimale Arbeitsbereich indirekter flammenspektrophotometrischer Analysenverfahren mit vorausgehender Fällungsreaktion. Mikrochim. Acta (Wien), 462 (1966). - 3. Handbuch zum Flammenphotometer Eppendorf, Eppendorf Gerätẹbau, Hamburg (1962). 4. KaISER, H., Z. analyt. Chem. 209, 1 (1965). - 5. HerrmanN, R. und C. Th. J. Alkemade, (übersetzt von P. T. GilberT), Flame Photometry Interscience Publishers, New York (1963). - 6. Menis, O., H. P. House und C. T. Rains, Analytic. Chem. 29, 76 (1957). - 7. Linder, A., Statistische Methoden, 3. Aufl. Birk- häuser Verlag, Basel-Stuttgart (1960). - 8. LANG, K., Biochem. Z. 290, 289 (1937). - 9. Schales, O. und S. S. Schales, J. biol. Chemistry 140, 879 (1941). - 10. Rausch, L. und H. Graul, Ärztl. Wschr. 4, 591 (1949). - 11. Stamm, D., Tagesschwankungen der Konzentration diagnostisch wichtiger Blutbestandteile. Habilitat., Med. Fak. Gießen (1966). - 12. Holleman-Wiberg, Lehrb. d. anorganischen Chemie, 47.-56. Auf. Walter de Gruyter \& Co., Berlin (1960). - 13. Frigr, F., Tüpfelanalyse, Bd. I. Akademische Verlagsgesellschaft mbH. Frankfurt/Main (1960).

Professor Dr. R. Herrmann 63 Gießen, Gaff kystr. 14

\section{Schnellbestimmung des Alkoholgehaltes im Blut und in anderen Körperflüssigkeiten}

\author{
Von E. VIDIC 1 ) \\ Aus dem Institut für gerichtliche und soziale Medizin der Freien Universität Berlin
}

(Direktor: Prof. Dr. med. W. Krauland)

(Eingegangen am 18. Januar 1967)

Für klinisch-diagnostische Zwecke sowie für wissenschaftliche Untersuchungen wurde ein Schnellverfahren zur Bestimmung des Alkoholgehaltes in Blut und anderen Körperflüssigkeiten entwickelt. In der beschriebenen, leicht zu bedienenden Glasapparatur kann die Bestimmung ohne besondere Einarbeitung in 20 Min. vorgenommen werden. Das Verfahren beruht auf der Verdampfung der Flüssigkeit in einem Luftstrom und Absorption der Dämpfe in Vanadinschwefelsäure. Bei der photometrischen Auswertung der Reaktion wird eine ausreichende Genauigkeit - entsprechend einer einfachen Standardabweichung von $0,05-0,08 \%$ - exzielt.

A rapid method for the determination of alcohol in blood and other body fluids was developed for clinical-diagnostic and for scientific studies. In the described, easily available glass apparatus, the determination can be carried out in 20 minutes without previous experience. The method is based on evaporation of the liquid in an air stream and adsorption of the vapour in vanadol-sulphuric acid. Photometric measurement of the reaction product provides a sufficiently accurate determination, with a standard deviation of $0.05-0.08 \%$.

Berichte und Statistiken der Kranken- und Heilanstalten sowie unsere Erfahrungen zeigen, daß Unfälle unter Alkoholeinwirkung immer häufiger werden. KRAMER (1) berichtete z. B. aus den Städt. Krankenanstalten Dortmund, da $B$ jeder siebente Patient betrunken zur Behandlung kam und daß $63 \%$ der Kopfverletzten alkoholisiert waren. Die Beurteilung der Kopfverletzungen wird bei dem Vorliegen einer Alkoholisierung erschwert, da durch sie zerebrale Symptome überdeckt werden können. Umgekehrt kann dabei oft der Grad der Alkoholisierung nicht erkannt werden, wodurch für den Patienten Gefahren erwachsen können. Auch in anderen Fällen, insbesondere bei der Einlieferung von unter Gift- oder Medikamentenwirkung stehenden Patienten, müssen oft Eingriffe und therapeutische Maßnahmen ohne Kenntnis des Grades einer evtl. vorhandenen Alkoholisierung vorgenommen werden, was zu Folgen für den Patienten führen kann. Eine rasche Kenntnis des Blutalkoholgehaltes kann ebenso bei der Auffindung

\footnotetext{
1) Auszugsweise vorgetragen und vorgeführt bei der Tagung der Deutschen Gesellschaft für gerichtliche Medizin in Freiburg/Br. am 8. Oktober 1966.
}

betrunkener, hilfloser Personen notwendig sein, um Gefahren abzuwenden. Der Alkoholisierungsgrad spielt auch bei der Beurteilung der Ergebnisse von Leichenöffnungen häufig eine nicht unbedeutende Rolle, so daß seine Bestimmung während der Vornahme der Sektion wesentlich zur Stellung der Diagnose beizutragen vermag. Nicht zuletzt kann auch bei wissenschaftlichen Untersuchungen sowie bei Trinkversuchen zur Ermittlung der Alkoholabbaugeschwindigkeit und bei der Feststellung von psychischen und physischen Ausfallerscheinungen eine rasche Orientierung über den Blutalkoholspiegel von erheblichem Vorteil sein. Die genannten, noch unvollständigen Hinweise auf die Bedeutung, die einer raschen Alkoholbestimmung zukommt, lassen erkennen, daß im Rahmen anderer diagnostischer Untersuchungen zweifellos auch ein echter Bedarf nach einem mit möglichst einfachen Mitteln durchführbaren und jederzeit einsatzbereiten Schnellverfahren besteht.

Bei dem derzeitigen Stande der Untersuchungstechnik sind die Laboratorien, die sich mit der routinemäßigen Bestimmung des Blutalkoholgehaltes befassen nicht in 
der Lage, ganz kurzfristig, etwa in 15 Min., das Resultat einer Blutalkoholbestimmung anzugeben. Die Zeitdauer, die zwischen dem Eintreffen der Blutprobe und der Mitteilung des festgestellten Blutalkoholgehaltes verstreicht, ist durch den Zeitaufwand für die angewandten Verfahren (meist Widmark- und ADH-Verfahren) sowie auch dadurch bedingt, da $\beta$ die Untersuchungen bei routinemäßiger Durchführung in großen Serien angesetzt und erst nach Beendigung der Analysen ausgewertet werden. Die zur automatischen Blutalkoholbestimmung dienenden Apparaturen, wie z. B. der AutoAnalyzer und der Gaschromatograph sind sehr kostspielige Geräte, die nur von Fachkräften nach längerer Einarbeitung bedient werden können. Außerdem sind diese recht komplizierten Apparate erst nach einer längeren Anlaufzeit einsatzbereit und liefern nur bei ständigem Betrieb zuverlässige Ergebnisse. Sie verlangen einen festen Standort und eine dauernde und sorgfältige Wartung und Überprüfung.

Um allen, an einer raschen Feststellung des Alkoholisierungsgrades einer Person interessierten Stellen die Möglichkeit zu geben, selbst Blutalkoholbestimmungen auszuführen, wurden Versuche zur Entwicklung eines mit einfachen apparativen Hilfsmitteln durchführbaren Schnellverfahrens unternommen. Als Nachweisverfahren war die bekannte Vanadinsäure-Methode (VIDIc 2-5) am besten geeignet, da es sich bei diesem Verfahren um eine photometrische Direktbestimmung des durch Umsetzung mit dem Alkohol entstandenen Reaktionsproduktes handelt, die von der Menge der vorgelegten Vanadinschwefelsäure unabhängig ist. Auf eine Beschleunigung dieser Reaktion abzielende Untersuchungen haben gezeigt, daß der aus einer kleinen Blutprobe in einem Luftstrom zusammen mit dem Wasser verdampfte Alkohol sehr rasch von heißer Vanadinschwefelsäure absorbiert und umgesetzt wird. Die weiteren Untersuchungen waren auf eine möglichst einfache und zweckmäßige Gestaltung der erforderlichen Apparatur, auf eine rasche Auswertbarkeit und eine möglichst geringe Fehlerstreuung der Analysenergebnisse gerichtet. Eine sehr befriedigende Genauigkeit wurde durch die Einstellung und exakte Konstanthaltung optimaler Werte für die Temperatur, die Luftgeschwindigkeit und Verdampfungsoberfläche der zu untersuchenden Flüssigkeitsprobe sowie für die Dauer der Reaktion erreicht. Die rasche Auswertbarkeit der am Photometer abgelesenen Extinktionen wurde durch eine einfache Vorschrift zur Herstellung einer Vanadinschwefelsäure mit hinreichend gleichbleibendem Oxydationspotential ermöglicht.

\section{Apparatur}

Bei einer großen Zahl von Untersuchungen hat sich eine Apparatur bewährt, deren wesentlichste Teile in der Abbildung 1 schematisch dargestellt sind ${ }^{1}$ ).

1) Die Apparatur ist als Gebrauchsmuster geschützt und wird als tragbares Koffergerät gebaut. Verfahren und apparative Einrichtungen sind zum Patent angemeldet.

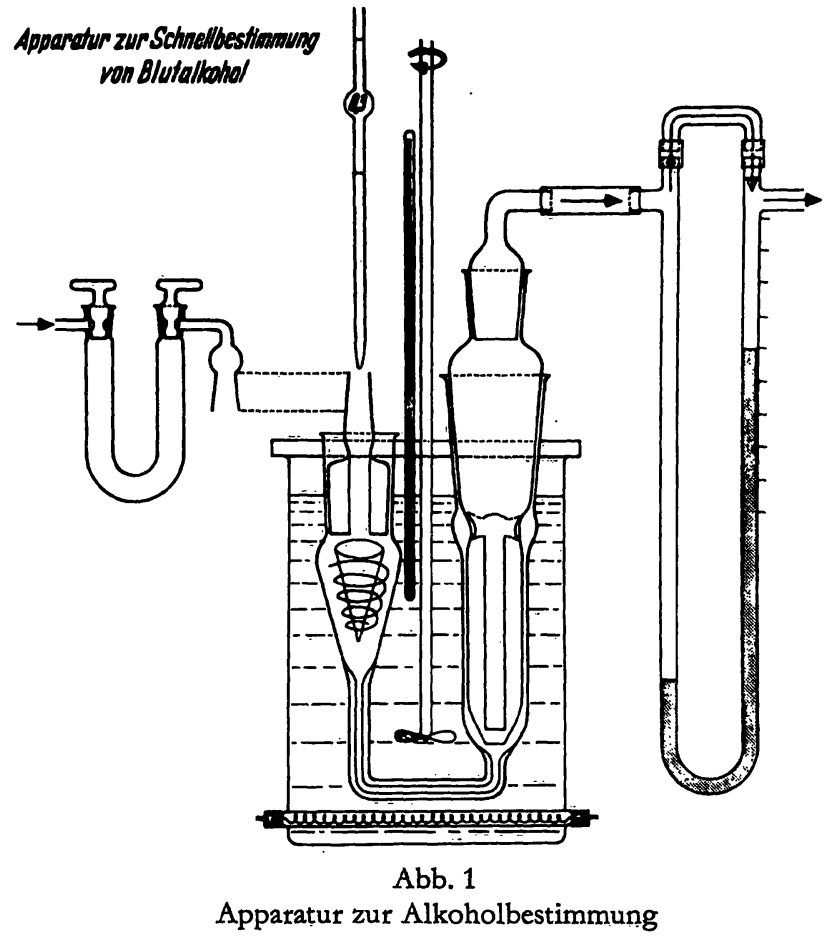

In einem mit Siliconöl gefüllten, elektrisch heizbaren Glasgefäß befindet sịch ein U-förmiges Glasgerät, das aus dem Verdampfer (links im Bild) und dem Reaktionsteilstück mit dem Einsatz für die Vanadinschwefelsäure besteht. Die Temperatur des Heizbades wird mittels Kontaktthermometer, Elektrohahn und Relais konstant auf $130^{\circ}$ gehalten. Ein kleines Rührwerk sorgt für eine gleichmäßige Wärmeverteilung. Im Verdampfer befindet sich eine Drahtspirale, die ein Faltenfilter aufnimmt. Der im Reaktionsteil in einem Schliff sitzende Einsatz wird außerhalb der Apparatur mit $5 \mathrm{~m} l$ Vanadinschwefelsäure beschickt. Durch die aufgeheizte Apparatur wird in Richtung der Pfeile ein mit einem Strömungsmesser eingestellter gleichbleibender Luftstrom hindurchgesaugt. Die in dem Einsatz befindliche Vanadinschwefelsäure wird dabei in $5 \mathrm{Min}$. auf die Reaktionstemperatur gebracht. Nun werden $0,3 \mathrm{~m} l$ des Blutserums oder einer anderen Körperflüssigkeit in einer mit 2 Marken versehenen geeichten Pipette abgemessen und nach Einführung der Pipette durch den Glasstopfen des Verdampfers auf das Filter auslaufen gelassen. Die Pipettenspitze wird an dem Filter abgestreift. Infolge des leichten Unterdrucks in der Apparatur entstehen hierbei keine Alkoholverluste. Der Luftstrom belädt sich mit dem verdampften Alkohol und streicht in kleinen Bläschen durch den Einsatz mit Vanadinschwefelsäure. In längstens 15 Min. ist der Alkohol aus der Probe quantitativ übergetrieben. Der Einsatz wird nun herausgenommen, kurz mit Wasser gekühlt und die Vanadinschwefelsäure mit dest. Wasser in ein zylindrisches Kolorimetergläschen oder einen Meßzylinder gespült und auf $20 \mathrm{~m} l$ aufgefüllt. Die Lichtabsorption der gebildeten blauen 4-wertigen Vanadinverbindung wird im Rotlicht (Absorptionsmaximum $750 \mathrm{~m} l l$ ) gemessen.

\section{Photometrie}

Die Genauigkeit der Bestimmungen wird größtenteils durch die Exaktheit der photometrischen Messung bestimmt. Bei etwas geringeren Ansprüchen an die Genauigkeit kann die in den zylindrischen Kolorimetergläsern verdünnte, noch warme Vanadinsäurelösung sogleich in einem Kolorimeter einfacherer Bauart (z. B. lichtelektrisches Kolorimeter der Fa. Lange, Berlin) gemessen werden. Bei Verwendung zylindrischer Gläser mit einem lichten Durchmesser von $24 \mathrm{~mm}$ beträgt die mittlere Standardabweichung $\pm 0,08 \%$, entsprechend einem $3 \mathrm{~s}$-Wert von $\pm 0,24 \%$ mit der hohen Wahr- 
scheinlichkeit von $99,73 \%$. Die Vertrauensgrenze von 3 s entspricht bereits einer außergewöhnlich hohen Genauigkeit, die über dem liegt, was üblicherweise unter dem Werturteil „mit an Sicherheit grenzender Wahrscheinlichkeit" verstanden wird. Die Eichlinie ist bei einer solchen Messung leicht gekrümmt, weil die Extinktionen mit zunehmendem Alkoholgehalt allmählich etwas stärker als linear ansteigen. Um eine rasche Feststellung des Promillegehaltes zu ermöglichen, wird das Kolorimeter mit einer Promille-Skala ausgerüstet, die aus der mittleren Eichkurve berechnet wird.

\section{Berechnung}

Mit planparallel geschliffenen Küvetten wird z. B. unter Verwendung des Lichtfilters $691 \mathrm{~m} \mu$ eine gerade, durch den Nullpunkt hindurchgehende Eichlinie erhalten, so $\mathrm{da} B$ sich der Promillegehalt der Probe durch einfache Multiplikation der abgelesenen Extinktion mit einem konstanten Faktor ergibt. Bei Verwendung eines geeigneten Photometers (z. B. Eppendorf-Photometer) kann der $3 s$-Wert auf $\pm 0,15 \%$ für die Einzelbestimmung gesenkt werden, wodurch die Genauigkeit des Widmark-Verfahrens erreicht wird. Der Umrechnungsfaktor der Extinktion auf den Promillegehalt beträgt dabei für $2 \mathrm{~cm}$ Schichtdicke z. B. 11,5, wenn die Vanadinsäure nach Vorschrift hergestellt wird. (Eine Extinktion von z. B. 0,200 ergibt somit einen Alkoholgehalt von 2,30\% . $^{\circ}$ ) Durch Anbringung einer PromilleSkala auf dem Photometer wird auch hier die Auswertung vereinfacht.

Für die Bestimmung des Blutalkoholgehaltes verwendet man zweckmäßigerweise Blutserum, weil dieses von dem Filter gleichmäßiger aufgesaugt wird, wodurch sich die Genauigkeit der Bestimmung erhöht. Der Promillegehalt im Vollblut wird erhalten, wenn man den Serumwert durch den konventionellen Faktor 1,2 dividiert. $\mathrm{Da}$ die Promillegehalte aber stets auf das Blutgewicht bezogen werden $(g \%)$, so ist auch noch durch das spezifische Gewicht des Serums 1,03 zu dividieren, wodurch sich ein Divisor von rund 1,24 ergibt. Leichenblut mit verhältnismäßig geringerem Plasmaanteil wird mit dem gleichen Volumen dest. Wasser verdünnt und der gefundene Gehalt mit 2 multipliziert und durch das spezifische Gewicht von Vollblut 1,055 dividiert. Urin und Liquor können unverdünnt untersucht werden.

\section{Herstellung der Vanadinschıefelsäure}

Die Oxydationsgeschwindigkeit des Alkohols durch Vanadinsäure ist, abgesehen von der Reaktionstemperatur und der Konzentration der Vanadinsäure, er- fahrungsgemäß in erheblichem Maße von der Art der Herstellung der Vanadinsäurelösung, also von der Konstitution der entsprechenden Vanadinsäuren abhängig. Es konnten aber Bedingungen für die Herstellung einer Vanadinsäurelösung mit gleichbleibendem Oxydationspotential gefunden werden, so daß jede vorschriftsmäßig hergestellte Vanadinsäurelösung stets die gleiche Eichkurve liefert. Die Vanadinsäurelösung wird aus einer wäßrigen Aufschlämmung von Vanadin-5Oxyd durch Vermischen mit konzentrierter Schwefelsäure in einfacher Weise nach der folgenden Vorschrift hergestellt:

In einem $300 \mathrm{~m} /$-Erlenmeyerkolben werden $3,4 \mathrm{~g}$ Vanadin-5-Oxyd, $\mathrm{V}_{2} \mathrm{O}_{5}(\mathrm{Fa}$. Merck) eingewogen und dann $30 \mathrm{ml}$ dest. Wasser zugesetzt. Zu dem Kolbeninhalt, der mit einem Magnetrührwerk gemischt wird, werden $170 \mathrm{~m} l$ konz. Schwefelsäure (p. a., $d=1,84$ ) aus einem zylindrischen Scheidetrichter zulaufen gelassen. Die Geschwindigkeit des Schwefelsäurezulaufes wird so bemessen, daß die Gesamtmenge in $4 \mathrm{Min}$. in den Kolben gelangt. $\mathrm{Zu}$ diesem Zwecke sind auf dem Scheidetrichter Marken angebracht, die das Schwefelsäurevolumen in vier gleiche Teile aufteilen. Infolge der bei der Verdünnung der Schwefelsäure auftretenden Enwärmung geht das Vanadinpentoxyd bei gleichmäßiger Rührung leicht in Lösung. Die Vanadinschwefelsäure läßt man in dem mit einem Schliffstopfen verschlossenen Kolben bei Zimmertemperatur abkühlen. Werden Zutritt von Feuchtigkeit und intensiver Lichteinfall verhindert, dann bleibt das Oxydationspotential der Vanadinschwefelsäure mindestens 1 Woche konstant. Bei einer Lagerzeit von 2-3 Wochen kann eine geringfügige Änderung des Oxydationspotentials (meist leichter Anstieg) eintreten, die jedoch für die meisten Ansprüche ohne Bedeutung ist.

Abschließend sei bemerkt, daß nicht beabsichtigt ist, die Schnellmethode allgemein für Blutalkohollaboratorien zu empfehlen, die laufend eine große Zahl von Routineuntersuchungen durchführen. Die Schnellbestimmung kann aber, infolge ihrer ausreichenden Genauigkeit überall da zur Anwendung kommen, wo unregelmäßig eine kleinere Zahl von Blutalkoholanalysen vorzunehmen ist, also z. B. in den eingangs erwähnten Fällen. Die Zuverlässigkeit, die dem Schnellverfahren auch in ungeübten Händen eigen ist, gestattet es, in der Mehrzahl der Fälle auf Zwei- oder Dreifachbestimmungen zu verzichten. Bei Verwendung eines geeigneten Photometers (z. B. Fa. Eppendorf oder Zeiss) kann schon mit einer Einzelbestimmung die Genauigkeit des WidmarkVerfahrens erreicht werden. Mit der einfachen, hier beschriebenen Apparatur können 3 Bestimmungen pro Stunde vorgenommen werden. Wenn zeitweise, infolge Häufung der Fälle, eine höhere stündliche Leistung erforderlich ist, kann der Analysendurchsatz durch Einbau von 2 oder 3 Verdampfern und Reaktionsteilen in ein Heizbad entsprechend gesteigert werden.

\section{Literatur}

1. Kramer, G., Med. Welt (N. V.) 17, 184 (1966). - 2. Vidic, E., Arzneimittel-Forsch., Aulendorf 4, 411 (1954). - 3. Vidrc, E.,
Atzneimittel-Forsch., Aulendorf 4, 506 (1954). - 4. VIDrc, E., Blutalkohol 2, 76 (1963). - 5. Vidic, E., Blutalkohol 2, 436 (1964). 